\title{
Search for a Metallicity Spread in the Multiple Population Large Magellanic Cloud Cluster NGC 1978
}

\author{
Andrés E. Piatti ${ }^{1,2}$ (1) and Jeremy Bailin ${ }^{3,4}$ (1) \\ ${ }^{1}$ Consejo Nacional de Investigaciones Científicas y Técnicas, Godoy Cruz 2290, C1425FQB, Buenos Aires, Argentina; andres@oac.unc.edu.ar \\ ${ }_{2}$ Observatorio Astronómico de Córdoba, Laprida 854, 5000, Córdoba, Argentina \\ ${ }^{3}$ Department of Physics and Astronomy, University of Alabama, Box 870324, Tuscaloosa, AL 35487-0324, USA \\ ${ }^{4}$ Steward Observatory, University of Arizona, 933 N Cherry Avenue, Tucson, AZ 85721-0065, USA \\ Received 2018 October 24; revised 2018 November 29; accepted 2018 December 1; published 2019 January 14
}

\begin{abstract}
We report on the spread of $[\mathrm{Fe} / \mathrm{H}]$ values in the massive Large Magellanic Cloud cluster NGC 1978, recently confirmed to harbor multiple populations of nearly the same age. We used accurate Strömgren photometry of carefully selected cluster red giant branch stars along with a high-dispersion spectroscopy-based calibration of the metallicity-sensitive index $m_{1}$. Once we accounted for the photometry quality, assessed from extensive artificial star tests to trace the photometric uncertainties as a function of the position of the cluster's center as well as the stellar brightness, and those from the metallicity calibration, we found that NGC 1978 exhibits a small metallicity spread of 0.035 dex $( \pm 0.019-0.023)$, depending on whether stars with individual $\sigma[\mathrm{Fe} / \mathrm{H}] \leqslant 0.15$ dex or those located in the cluster's outer areas are considered. Such a spread in $[\mathrm{Fe} / \mathrm{H}]$ is consistent with a cluster formation model with self-enrichment, if mass loss higher than $90 \%$ due to stellar evolutionary and galactic tidal effects is assumed. Nevertheless, scenarios in which the apparent $[\mathrm{Fe} / \mathrm{H}]$ variation reflects $\mathrm{CN}$ abundance anomalies or less extreme mass-loss models with environmentally dependent self-enrichment should not be ruled out.
\end{abstract}

Key words: galaxies: individual (LMC) - galaxies: star clusters: general

\section{Introduction}

A number of theoretical models have recently proposed different scenarios to explain anomalies in the abundances of several chemical elements in massive clusters harboring multiple populations (MPs; see, e.g., Bekki \& Tsujimoto 2016; Bekki 2017; Bailin 2018a; Gieles et al. 2018; Kim \& Lee 2018, and references therein). They have mainly been stimulated by observational results of anticorrelations between chemical abundances of light elements. Some of the models also suggest mechanisms to obtain intrinsic $[\mathrm{Fe} / \mathrm{H}]$ spreads $>0.05 \mathrm{dex}$, as observed at the present time in eight Milky Way (MW) old globular clusters (Marino et al. 2015; Lim et al. 2017).

NGC 1978 is a massive $\left(\sim 3 \times 10^{5} M_{\odot}\right.$ Piatti \& Bastian 2016a) intermediate-age Large Magellanic Cloud (LMC) cluster (2 Gyr Milone et al. 2009; Goudfrooij et al. 2014) with a mean metallicity of $[\mathrm{Fe} / \mathrm{H}]=-0.38 \pm 0.05$ dex (Ferraro et al. 2006). Lederer et al. (2009) and Davidge (2018) have discussed its puzzling metallicity pattern overviewing previous studies carried out on this object, although they did not discuss any possible Fe-abundance spread. NGC 1978 has recently been found to harbor MPs based on $[\mathrm{N} / \mathrm{Fe}]$ variations among red giant branch (RGB) stars (Martocchia et al. 2018a), a feature also seen in almost all MW globular clusters with MPs. Hence, the authors concluded that there must be a common formation and evolutionary process for massive clusters $\left(\gtrsim 10^{5} M_{\odot}\right)$, regardless of their ages. Additionally, NGC 1978's MPs are of nearly the same age (Martocchia et al. 2018b), so their abundance dispersions have somehow originated concurrently.

In this paper we report on the magnitude of the intrinsic [Fe/H] spread in NGC 1978. In order to measure it, we made use of the Strömgren photometric data set described in Section 2. We performed a careful selection of cluster RGB members and estimated individual $[\mathrm{Fe} / \mathrm{H}]$ values from a high-dispersion spectroscopy-based calibration of the metallicity-sensitive index $m_{1}$, as detailed in Section 3. In Section 4 we analyze the metallicity estimates in the light of a maximum likelihood approach and discuss the results in the context of selfenrichment cluster formation models. Finally, Section 5 summarizes the main conclusions of this work.

\section{Strömgren Photometry Data Set}

We downloaded publicly available Strömgren $v b y$ images from the National Optical Astronomy Observatory (NOAO) Science Data Management (SDM) Archives. ${ }^{5}$ They were obtained, along with suitable calibration and standard field images, as part of an observational program aiming at studying the chemical evolution of the LMC from clusters and field stars (program ID: SO2008B-0917, PI: Pietrzynski). The images were obtained on the night of 2008 January 18 with the SOAR Optical Imager (SOI) mounted on the $4.1 \mathrm{~m}$ Southern Astrophysical Research (SOAR) telescope $(\mathrm{FOV}=5 ! 25 \times 5 ! 25$, scale $=0$ !' $154 / \mathrm{px})$, and are of excellent quality (typical FWHM $\sim 0$ "' 6 ). They consist of one exposure of 350,140 , and $90 \mathrm{~s}$ in the $v, b$, and $y$ filters, respectively. In order to properly process them, we followed the SOI's pipeline guidance described at http://www.ctio.noao.edu/ soar/content/soar-optical-imager-soi.

To standardize our photometry we measured instrumental vby magnitudes of the standard stars HD 64, HD 3417, HD 12756, HD 22610, TYC 7583-1622-1, TYC 8104-969-1, HD 57568, and HD 58489 (Hauck \& Mermilliod 1998; Paunzen 2015). The stars were observed at airmass between 1.05 and 2.83 along the whole night. Each star was observed twice at a given airmass, in order to place it in each of the two CCDs used by SOI. We then performed fits of the expressions:

\footnotetext{
5 http//www.noao.edu/sdm/archives.php
} 


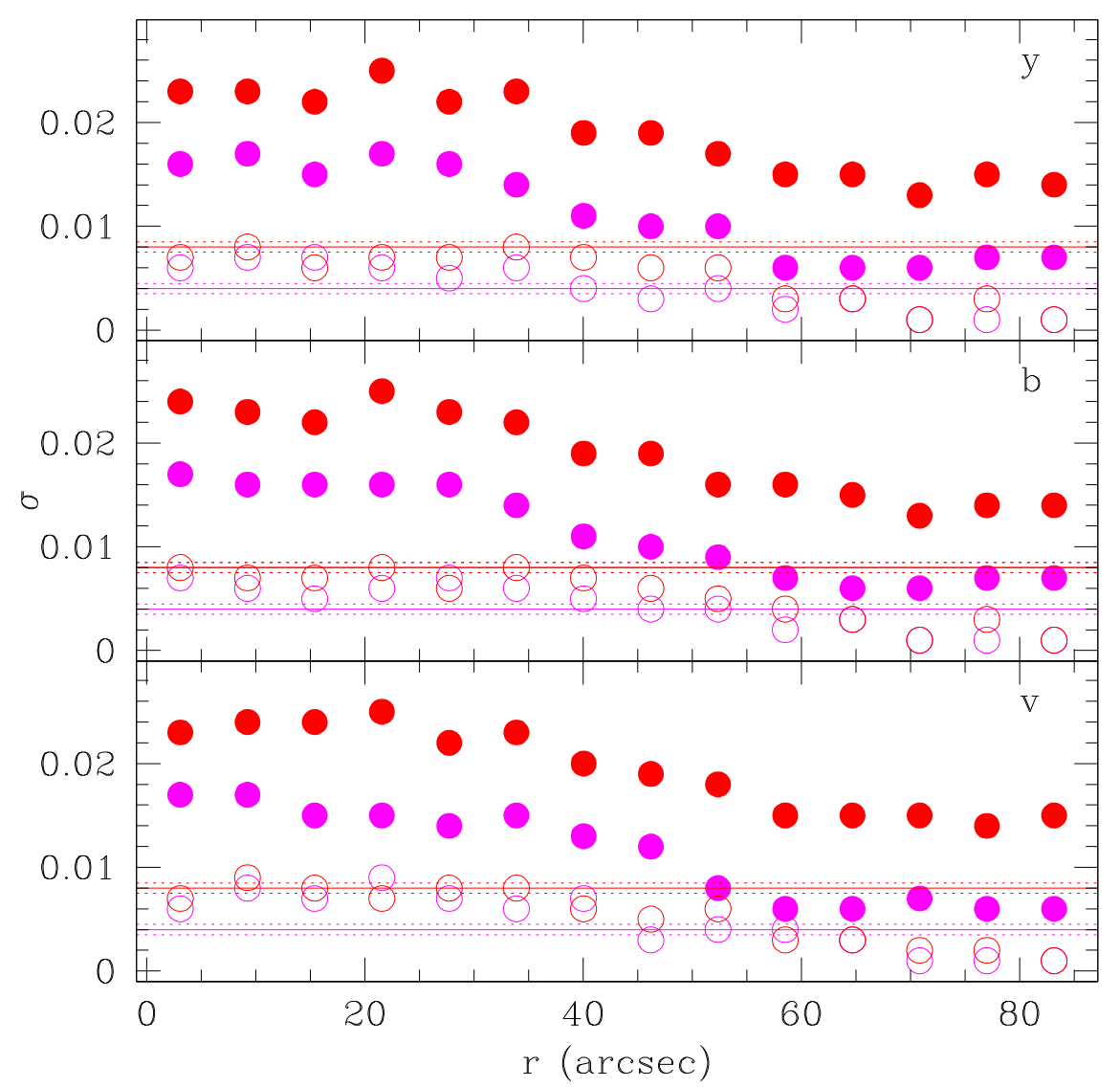

Figure 1. Photometric error estimates for the $v b y$ filters as a function of the distance to the cluster center. Magenta and red symbols are for $V=16.5$ and 18.5 mag, respectively. Solid and dotted lines represent the errors returned from applying the PSF models. Open and filled circles are the difference between the mean input and output magnitudes of the thousand realizations of artificial star tests and their rms errors, respectively.

Table 1

Strömgren Transformation Coefficients

\begin{tabular}{lccccr}
\hline \hline Filter & coef $_{1}$ & coef $_{2}$ & coef $_{3}$ & coef $_{4}$ & rms \\
\hline$v$ & $\# 1$ & $1.255 \pm 0.023$ & $0.276 \pm 0.012$ & $1.823 \pm 0.087$ & $1.148 \pm 0.095$ \\
& $\# 2$ & $1.264 \pm 0.027$ & $0.290 \pm 0.015$ & $1.822 \pm 0.103$ & $1.135 \pm 0.111$ \\
& full & $1.259 \pm 0.023$ & $0.280 \pm 0.012$ & $1.823 \pm 0.087$ & 0.009 \\
& & & & 0.011 \\
$b$ & $\# 1$ & $0.998 \pm 0.016$ & $0.181 \pm 0.008$ & $0.940 \pm 0.027$ & 0.009 \\
& $\# 2$ & $1.009 \pm 0.010$ & $0.188 \pm 0.005$ & $0.928 \pm 0.016$ & 0.009 \\
& full & $1.003 \pm 0.010$ & $0.184 \pm 0.005$ & $0.934 \pm 0.016$ & 0.006 \\
$y$ & & & & \\
& $\# 1$ & $0.982 \pm 0.024$ & $0.131 \pm 0.013$ & $-0.024 \pm 0.041$ & \\
& $\# 2$ & $0.999 \pm 0.015$ & $0.131 \pm 0.008$ & $-0.007 \pm 0.025$ & 0.014 \\
& full & $0.990 \pm 0.015$ & $0.131 \pm 0.008$ & $-0.015 \pm 0.025$ & 0.009 \\
& & & & 0.009 \\
\hline
\end{tabular}

$$
\begin{aligned}
& v=v_{1}+V_{\text {std }}+v_{2} \times X_{v}+v_{3} \times(b-y)_{\text {std }}+v_{4} \times m_{1 \mathrm{std}}, \\
& b=b_{1}+V_{\text {std }}+b_{2} \times X_{b}+b_{3} \times(b-y)_{\text {std }} \\
& y=y_{1}+V_{\text {std }}+y_{2} \times X_{y}+y_{3} \times(b-y)_{\text {std }}
\end{aligned}
$$

where $v_{i}, b_{i}$, and $y_{i}$ are the $i$ th fitted coefficients, and $X$ represents the effective airmass. Table 1 shows the resulting coefficients for stars measured in the two different CCDs, separately. As can be seen, there is an excellent agreement between the independent transformation coefficients from both CCDs. For this reason, we decided to use all the measured stars, regardless of their positions in SOI. The resulting coefficients are also listed in Table 1.

We obtained instrumental vby magnitudes of stars located in the field of NGC 1978 using the stand-alone versions of
DAOPHOT, ALLSTAR, DAOMATCH, and DAOMASTER routine packages (Stetson et al. 1990). The magnitudes were derived from point-spread function (PSF) fits performed using previously generated spatially quadratically varying PSFs. These PSFs were modeled from a sample of nearly 100 interactively selected stars distributed throughout the image, previously cleaned from fainter contaminating neighboring stars using a preliminary PSF built with nearly 40 relatively bright, well-isolated stars. Once we applied the resulting PSF to an image, we took advantage of the subtracted image to identify new fainter sources which were added to the final photometric catalog. In each of the three iterations performed, we did the PSF photometry for the whole sample of identified 

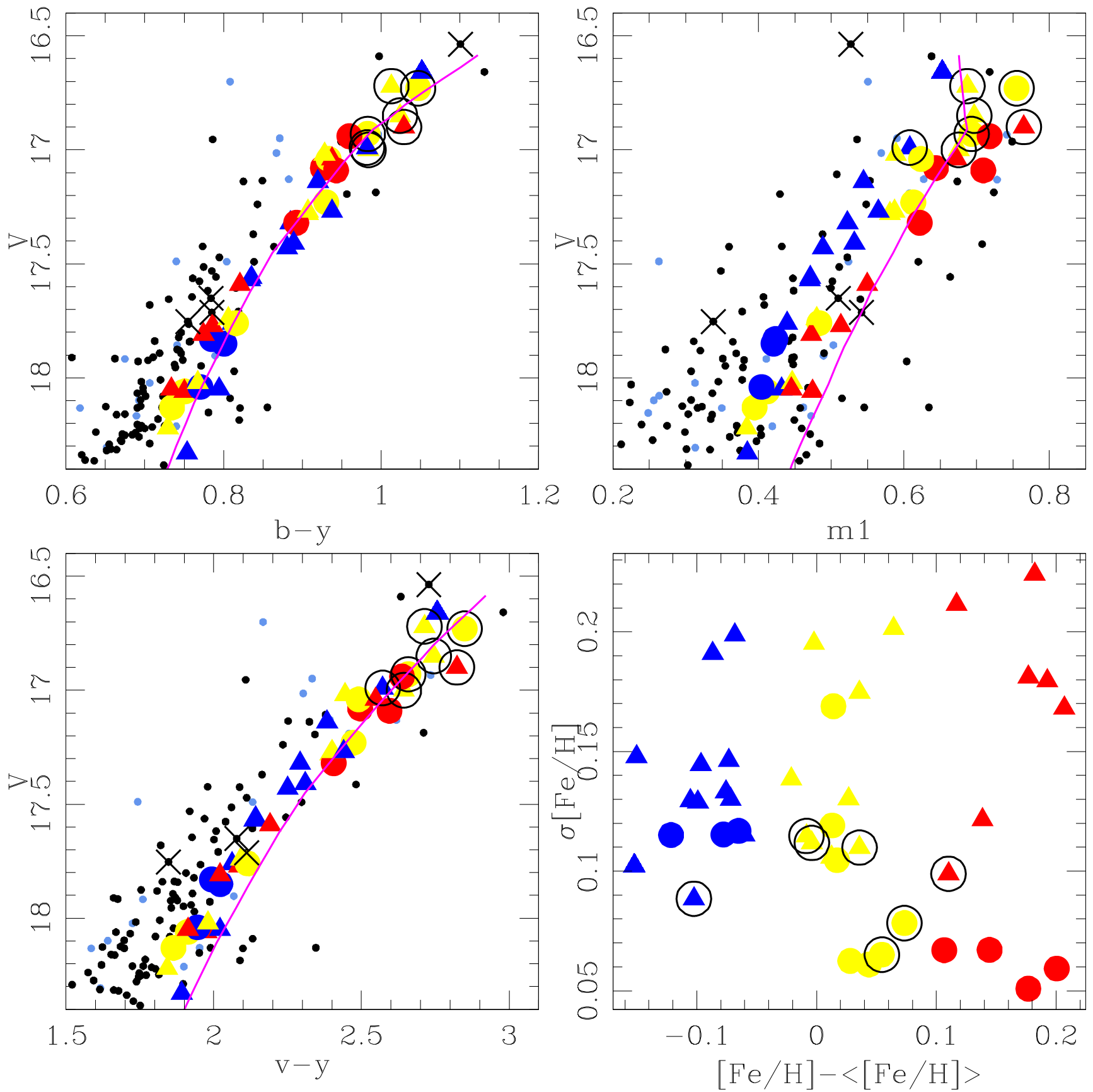

Figure 2. Three different CMDs for measured stars distributed within the cluster region (black small filled circles) and those selected for the metallicity analysis (large filled circles and triangles, color coded by metallicity as illustrated in the bottom-right panel), and those for an equal cluster area star field (light-blue small filled circles). Crosses represent AGB stars measured in the cluster field (Kamath et al. 2010). Large filled triangles and circles correspond to stars located closer and farther than 50 arcsec from the cluster's center. The bottom-right panel shows the $[\mathrm{Fe} / \mathrm{H}]$ difference for the selected stars with respect to the mean cluster value, depicted vs. the derived $[\mathrm{Fe} / \mathrm{H}]$ errors. Stars with big black open circles are those in common with Ferraro et al. (2006).

sources. Finally, we transformed the instrumental magnitudes into the Strömgren photometric system using the coefficients listed in Table 1. Figure 1 illustrates the mean and standard dispersion (solid and dotted lines) of the resulting photometric errors for two different magnitude levels, namely $V=16.5$ mag and $18.5 \mathrm{mag}$, respectively. These magnitudes roughly correspond to the upper and lower limits of the cluster RGBs used in this work (see Figure 2). Note that these errors do not increase as functions of radius, revealing that the PSF model is accurate. Table 2 shows a portion of the resulting photometric catalog.
We rigorously examined the quality of our photometry aiming at obtaining robust estimates of the photometric errors. In doing that, we performed the widely accepted artificial star tests (see, e.g., Piatti \& Bastian 2016b; Piatti \& Cole 2017; Piatti \& Mackey 2018, and references therein) by using the stand-alone ADDSTAR program in the DAOPHOT package (Stetson et al. 1990) to add synthetic stars, generated bearing in mind the color and magnitude distributions of the stars in the color-magnitude diagram (CMD) as well as the cluster radial stellar density profile. We added a number of stars equivalent to $\sim 5 \%$ of the measured stars in order to avoid in the synthetic 
Table 2

Strömgren Photometry of Stars in the Field of NGC 1978

\begin{tabular}{|c|c|c|c|c|c|c|c|c|c|}
\hline Star & $\begin{array}{l}\text { R.A. } \\
\text { (deg) }\end{array}$ & $\begin{array}{l}\text { Decl. } \\
\text { (deg) }\end{array}$ & $\begin{array}{c}r^{\mathrm{a}} \\
(\operatorname{arcsec})\end{array}$ & $\begin{array}{c}V \\
(\mathrm{mag})\end{array}$ & $\begin{array}{c}\sigma(V) \\
(\mathrm{mag})\end{array}$ & $\begin{array}{l}b-y \\
(\mathrm{mag})\end{array}$ & $\begin{array}{c}\sigma(b-y) \\
(\mathrm{mag})\end{array}$ & $\begin{array}{c}m_{1} \\
(\mathrm{mag})\end{array}$ & $\begin{array}{l}\sigma\left(m_{1}\right) \\
(\mathrm{mag})\end{array}$ \\
\hline$\cdots$ & $\cdots$ & $\cdots$ & $\cdots$ & $\cdots$ & $\cdots$ & $\cdots$ & $\cdots$ & $\ldots$ & \\
\hline 832 & 82.180946 & -66.205498 & 110.2 & 18.081 & 0.016 & 0.699 & 0.021 & 0.458 & 0.034 \\
\hline 833 & 82.208344 & -66.215805 & 79.0 & 19.133 & 0.013 & 0.611 & 0.019 & 0.340 & 0.031 \\
\hline 834 & 82.148354 & -66.191719 & 169.0 & 18.132 & 0.008 & 0.618 & 0.012 & 0.350 & 0.019 \\
\hline$\cdots$ & $\cdots$ & $\cdots$ & $\cdots$ & $\cdots$ & $\cdots$ & $\cdots$ & $\cdots$ & $\cdots$ & \\
\hline
\end{tabular}

Note.

a Distance to the cluster center.

images significantly more crowding than in the original images. On the other hand, to avoid small number statistics in the artificial star analysis, we created a thousand different images for each original one. We used the option of entering the number of photons per ADU in order to properly add the Poisson noise to the star images.

We then repeated the same steps to obtain the photometry of the synthetic images as described above, i.e., performing three passes with the DAOPHOT/ALLSTAR routines. Figure 1 shows with open circles the difference between the mean input and output magnitudes for the thousand realizations of added synthetic stars-matched using the DAOMATCH and DAOMASTER tasks-for the magnitude levels $16.5 \mathrm{mag}$ and 18.5 mag, respectively. These differences show that we were able to recover reliable magnitudes along the whole range of distances. Nevertheless, as a conservative representative of the photometry uncertainties we adopted the rms errors of all artificial star tests (filled circles in Figure 1), that best reflect the crowding effects, as expected.

\section{Metallicity Estimates}

To estimate the metallicity of each star we used the calibration of giant stars based on both the metallicity-sensitive index $m_{1}$ and the $v-y$ color derived by Calamida et al. (2007). Particularly, we used the inverted expression, i.e., $[\mathrm{Fe} / \mathrm{H}]$ as a function of the reddening free $m_{1 o}$ and $(v-y)_{o}$ colors (Frank et al. 2015, Equation (3)), as follows:

$$
[\mathrm{Fe} / \mathrm{H}]=\frac{m_{1_{o}}+a_{1}(v-y)_{o}+a_{2}}{a_{3}(v-y)_{o}+a_{4}},
$$

where $a_{1}=-0.521 \pm 0.001, \quad a_{2}=0.309, \quad a_{3}=0.159 \pm$ 0.001 , and $a_{4}=-0.090 \pm 0.002$, respectively. In doing that, we first adopted the $E(B-V)$ value used by Martocchia et al. $(2018 \mathrm{a}, 2018 \mathrm{~b})$ and the $E(X) / E(B-V)$ ratios given by Crawford \& Mandwewala (1976) to obtain $E\left(m_{1}\right)$ and $E(v-y)$ color excesses, from which we corrected $m_{1}$ and $v-y$ for the effects of reddening. Because the Calamida et al. (2007) calibration is based on old globular clusters, and NGC 1978 is much younger, the derived $[\mathrm{Fe} / \mathrm{H}]$ distribution result is centered on a more metal-poor value $([\mathrm{Fe} / \mathrm{H}]=$ $-1.0 \mathrm{dex})$ than the cluster mean metallicity $([\mathrm{Fe} / \mathrm{H}]=-0.38$ dex) (Ferraro et al. 2006) due to the well-known age effect on metallicities obtained from the cluster RGB (see, e.g., Geisler et al. 2003; Ordoñez \& Sarajedini 2015). Nevertheless, that constant offset does not have any effects on the subsequent analysis, because we are interested only in the spread of
$[\mathrm{Fe} / \mathrm{H}]$ values with respect to the mean value. Because the MPs of NGC 1978 have essentially identical ages (Martocchia et al. $2018 b$ ), the difference in ages will not artificially inflate the measured $[\mathrm{Fe} / \mathrm{H}]$ dispersion.

In order to estimate the uncertainties of the individual $[\mathrm{Fe} / \mathrm{H}]$ values we thoroughly performed a full analytical propagation of errors, including those on the calibration coefficients of Equation (1), as follows:

$$
\begin{gathered}
\sigma([\mathrm{Fe} / \mathrm{H}])^{2}=\left(\frac{\partial[\mathrm{Fe} / \mathrm{H}]}{\partial a_{1}} \sigma\left(a_{1}\right)\right)^{2}+\left(\frac{\partial[\mathrm{Fe} / \mathrm{H}]}{\partial a_{2}} \sigma\left(a_{2}\right)\right)^{2} \\
+\left(\frac{\partial[\mathrm{Fe} / \mathrm{H}]}{\partial a_{3}} \sigma\left(a_{3}\right)\right)^{2}+\left(\frac{\partial[\mathrm{Fe} / \mathrm{H}]}{\partial a_{4}} \sigma\left(a_{4}\right)\right)^{2} \\
+\left(\frac{\partial[\mathrm{Fe} / \mathrm{H}]}{\partial m_{1_{o}}} \sigma\left(m_{1_{o}}\right)\right)^{2}+\left(\frac{\partial[\mathrm{Fe} / \mathrm{H}]}{\partial(v-y)_{o}} \sigma\left((v-y)_{o}\right)\right)^{2} \\
\sigma([\mathrm{Fe} / \mathrm{H}])^{2}=\left(\frac{0.001(v-y)_{o}}{c}\right)^{2}+\left(\frac{0.001[\mathrm{Fe} / \mathrm{H}](v-y)_{o}}{c}\right)^{2} \\
+\left(\frac{0.002[\mathrm{Fe} / \mathrm{H}]}{c}\right)^{2}+\left(\frac{\sigma\left(m_{1_{o}}\right)}{c}\right)^{2} \\
+\left(\frac{\left(-0.521 c-0.159\left(m_{1_{o}}+0.309-0.521(v-y)_{o}\right) \sigma\left((v-y)_{o}\right)\right.}{c^{2}}\right)^{2}
\end{gathered}
$$

where $c=a_{3}(v-y)_{o}+a_{4}$, and $\sigma\left(m_{1_{o}}\right)$ and $\sigma\left((v-y)_{o}\right)$ are the photometric errors in $m_{1_{o}}$ and $(v-y)_{o}$, respectively, according to the position of the stars with respect to the cluster's center (see Figure 1) and the expressions:

$$
\begin{gathered}
(v-y)_{o}=v-y-1.67 \times 0.74 E(B-V), \\
\sigma\left((v-y)_{o}\right)^{2}=\sigma(v)^{2}+\sigma(y)^{2}+ \\
(1.67 \times 0.74 \sigma(E(B-V)))^{2}
\end{gathered}
$$

and

$$
\begin{aligned}
& m_{1 o}=(v-b)-(b-y)+0.33 \times 0.74 E(B-V) \\
& \sigma\left(m_{1 o}\right)^{2}=\sigma(v)^{2}+4 \sigma(b)^{2}+ \\
& \sigma(y)^{2}+(0.33 \times 0.74 \sigma(E(B-V)))^{2}
\end{aligned}
$$

The RGB star sample we finally kept to investigate any metallicity variation in NGC 1978 was carefully selected following these criteria:

1. The stars are located inside the cluster tidal radius (Werchan \& Zaritsky 2011; Piatti \& Bastian 2016a).

2. Stars located beyond 0.05 mag in $b-y$ from the cluster RGB ridge line in the $V$ versus $b-y$ CMD were discarded. Note that the cluster RGB in the $V$ versus 
$b-y$ CMD is not strongly affected by metallicity, so it results in a narrow star sequence. We only considered RGB stars brighter than the cluster red clump/horizontal branch.

According to Kamath et al. (2010), NGC 1978 hosts a nonzero population of AGB stars, which can add a contamination of up to $10 \%$ of the stellar sample as analyzed here. To assess their influence on the metallicity spread, we first consulted a set of Padova isochrones (Bressan et al. 2012), available in the Strömgren system, with ages and metallicities tailored to NGC 1978. We then created random samples of stars matching the observed numbers. and designed them to lie either on the theoretical RGB or AGB tracks; next, we computed their metallicities from the above calibrations. Despite the inherent mono-metallicity of the isochrones, this procedure introduces a standard deviation on the order of 0.04 dex from theoretical considerations for both classes of stars. Moreover, metallicities derived from AGB stars are on average lower by merely $0.02 \mathrm{dex}$, so that the inclusion of a minor contamination in our presumed RGB sample would not lead to a significant inflation of the intrinsic dispersion if they were misclassified as RGB stars. For instance, from $V=16.6$ down to 17.07 out of nine selected stars are RGB stars observed from high-dispersion spectroscopy (Ferraro et al. 2006), that expand the readily visible range of metallicities in NGC 1978. From $V=17.5$ down to 18.4 , the sequence of AGB stars is clearly distinguished from that of RGB stars. Moreover, we note that, as AGB stars are warmer and less massive than RGB stars, their metallicities should follow different relations of the Calamida et al. (2007) calibration that was used here, also due to the different strength of the molecular bands, with weaker $\mathrm{CN}$ - and $\mathrm{CH}$-bands. Indeed, four AGB stars found in our sample have $[\mathrm{Fe} / \mathrm{H}]$ values much more different that those shown by RGB stars.

The combination of these criteria allow us to remove almost all field stars, as well as cluster AGBs. Nevertheless, we counted the number of field stars distributed in several different regions around the cluster and of an equal cluster area and found on average that they represent less than $2 \%$ with respect to the number of selected stars located along the delineated RGB ridge line in the $V$ versus $b-y$ CMD. Figure 2 illustrates the distribution of the selected stars in three different CMDs as well as their derived $[\mathrm{Fe} / \mathrm{H}]$ values and uncertainties (note that the $V$ versus $v-y$ panel does not provide independent information, but is included for completeness). For comparison purposes we used three arbitrary metallicity-based color ranges. As can be seen, selected stars showing no readily visible color spread in the $V$ versus $b-y$ CMD, do exhibit a clear broadness in the $V$ versus $m_{1} \mathrm{CMD}$, which is tightly related to the variation in the cluster metallicity content. In order to illustrate the impact of the adopted photometric errors in the metallicity uncertainties, which depend on both the brightness and the position of a star in the cluster field, we drew with large filled triangles and circles stars located closer and farther than 50 arcsec from the cluster's center, respectively. For completeness, we included all the stars measured within the cluster area and those for a comparison field region with black and lightblue filled circles, respectively. Four measured AGB stars out of a total of 12 cataloged by Kamath et al. (2010) are drawn with a cross. The magenta line is the theoretical isochrone of Bressan et al. (2012) for an age of $2 \mathrm{Gyr}$ and a metallicity of $[\mathrm{Fe} / \mathrm{H}]=-0.40 \mathrm{dex}$, shifted by the cluster color excess and distance modulus. As can be seen, the combination of accurate photometry (see Table 1 and Figure 1) and an accurate metallicity calibration (see Equation (1)) result in an advantageous tool for estimating cluster RGB stars' metallicities with uncertainties that, in the case of the brightest objects, are of the same order as those expected from high-dispersion spectroscopy.

\section{Analysis and Discussion}

By using the selected stars, we observe a spread in the mean $[\mathrm{Fe} / \mathrm{H}]$ values derived from Calamida et al.'s (2007) $m_{1}$ index calibration of $\sim 0.35$ dex (see the bottom-right panel of Figure 2). Nevertheless, in order to quantify the real spread in the derived $[\mathrm{Fe} / \mathrm{H}]$ values, we used the well-known maximum likelihood approach by optimizing the probability $\mathcal{L}$ that the sample of selected stars with metallicities $[\mathrm{Fe} / \mathrm{H}]_{i}$ and errors $\sigma_{i}$ are drawn from a population with mean $\langle[\mathrm{Fe} / \mathrm{H}]\rangle$ and dispersion $W$ (e.g., Pryor \& Meylan 1993; Walker et al. 2006), as follows:

$$
\mathcal{L}=\prod_{i=1}^{N}\left(2 \pi\left(\sigma_{i}^{2}+W^{2}\right)\right)^{-\frac{1}{2}} \exp \left(-\frac{\left([\mathrm{Fe} / \mathrm{H}]_{i}-\langle[\mathrm{Fe} / \mathrm{H}]\rangle\right)^{2}}{2\left(\sigma_{i}^{2}+W^{2}\right)}\right),
$$

where the errors on the mean and dispersion were computed from the respective covariance matrices. If we constrained the sample of selected stars to those with $\sigma[\mathrm{Fe} / \mathrm{H}]<0.15 \mathrm{dex}$, we would obtain $W=0.035 \pm 0.019 \mathrm{dex}$, while for stars located between $r=50$ and 85 arcsec, we would derive $W=0.035 \pm 0.023$ dex, respectively. By using stars with $\sigma$ $[\mathrm{Fe} / \mathrm{H}]>0.15 \mathrm{dex}$, any marginal dispersion is blurred. We note that an underestimate of the photometric errors would result in a correspondingly smaller intrinsic spread. For instance, a $20 \%$ increase in the adopted errors reduces the intrinsic spread to $W=0.025 \pm 0.020$, while a $50 \%$ increase leads to a null $W$ value. This latter result is compatible with using stars with $\sigma[\mathrm{Fe} / \mathrm{H}]>0.15$ dex. As for the error in the mean $[\mathrm{Fe} / \mathrm{H}]$, we derived in all the adopted configurations $\sigma\langle[\mathrm{Fe} / \mathrm{H}]\rangle=0.020$ dex.

We also computed the mean and dispersion for seven stars in common with Ferraro et al. (2006; see Figure 3 and stars with a big black open circle in Figure 2) and obtained $\sigma\langle[\mathrm{Fe} / \mathrm{H}]\rangle=0.03$ dex and a null $W$ with an upper limit of $W \leqslant 0.05$ dex, while from Ferraro et al.'s (2006) Feabundances, we derived $\langle[\mathrm{Fe} / \mathrm{H}]\rangle=-0.43 \pm 0.06$ dex and a null $W$ with an upper limit of $W \leqslant 0.07 \mathrm{dex}$, respectively. Although the large uncertainties in Ferraro et al. (2006) preclude us from seeing a direct correlation between the present metallicities and those from the high-dispersion spectroscopy on a star-by-star basis, they are completely consistent. Since the seven stars in common do not span the whole observed $[\mathrm{Fe} / \mathrm{H}]$ range, a robust measurement of the small spread is not possible from this small subsample of stars using either abundance measurement; however, the upper limit derived from this subsample is consistent with the spread we measure from the full sample.

From the above results, we concluded that NGC 1978 exhibits a small spread in the $[\mathrm{Fe} / \mathrm{H}]$ values, as is the case of all the MW old globular clusters, with the exception of eight that have spreads in $[\mathrm{Fe} / \mathrm{H}]>0.05 \mathrm{dex}$ (Carretta et al. 2009; Willman \& Strader 2012; Marino et al. 2015). Nevertheless, because the $\mathrm{CN}$ absorption band at $4142 \AA$ is near to the effective wavelength of the $v$ filter, and hence could be measured by the $m_{1}$ index, the resulting intrinsic $[\mathrm{Fe} / \mathrm{H}]$ spread 


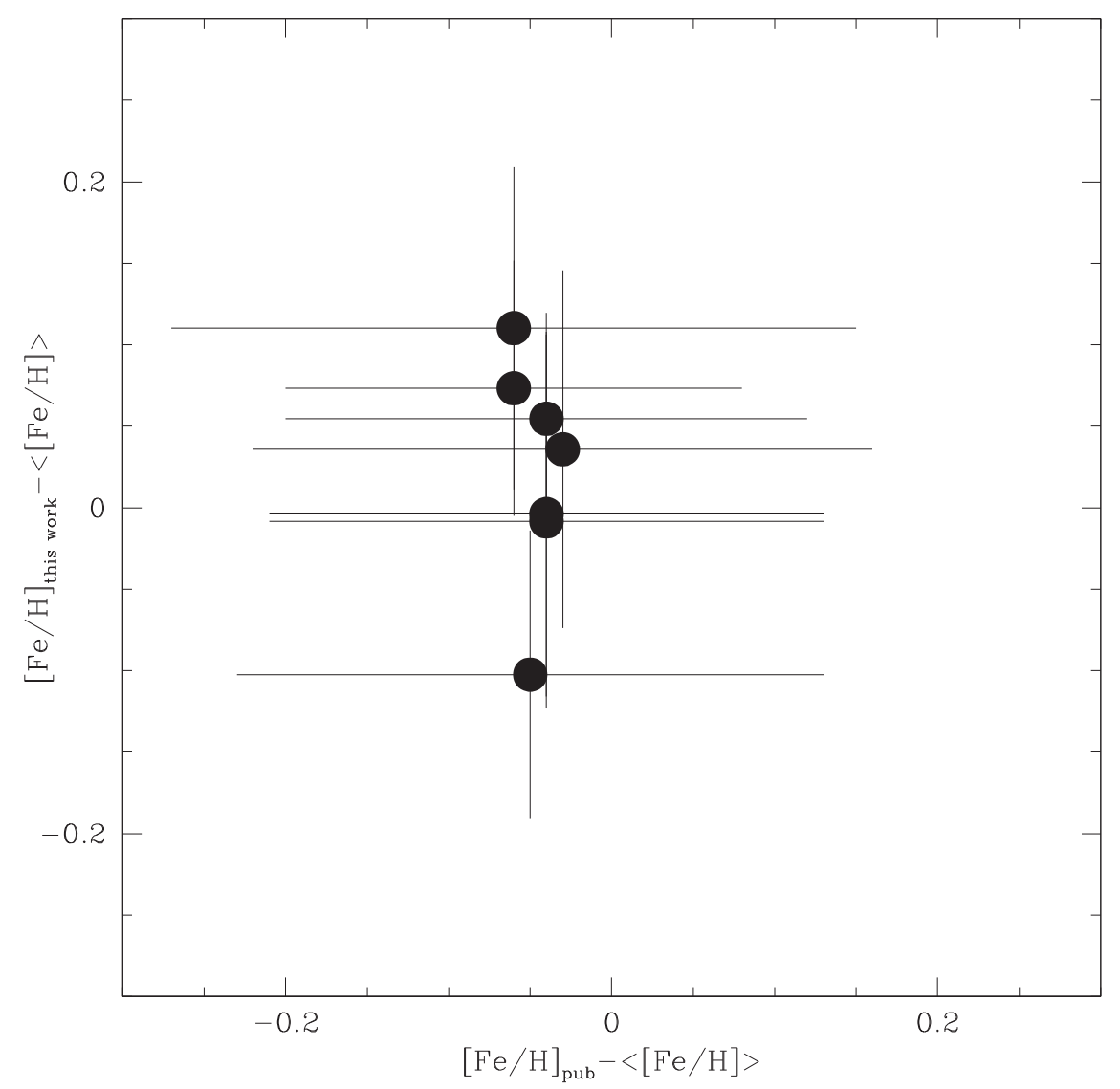

Figure 3. Comparison between Ferraro et al.'s (2006) metallicities $\left([\mathrm{Fe} / \mathrm{H}]_{\text {pub }}\right)$ and our values $\left([\mathrm{Fe} / \mathrm{H}]_{\text {this }}\right.$ work $)$.

could reflect $\mathrm{CN}$ variations. In such a case, we should interpret this small spread as reflecting the existence of MPs. However, there are a number of reasons to favor an interpretation with both light and heavy element abundance variations. First, the $m_{1}$ index was calibrated by Calamida et al. (2007) as a photometric proxy for the iron abundance. Indeed, Piatti \& Koch (2018) have recently obtained Strömgren-based [Fe/H] values for 10 ancient LMC globular clusters over a broad metallicity range $(-2.1 \leqslant[\mathrm{Fe} / \mathrm{H}](\mathrm{dex}) \leqslant-1.0)$ in excellent agreement with mostly high-dispersion spectroscopic values. Second, Lim et al. (2017) showed that old globular clusters with MPs show both light and heavy element abundance variations (see also Marino et al. 2015). Third, Piatti (2018) showed that accurate Strömgren photometry is able to unveil intermediate-age clusters harboring MPs.

\subsection{Self-enrichment Model}

Bailin (2018a) presented a model for clumpy self-enrichment in globular clusters, whereby core collapse supernova ejecta from high mass stars in one protocluster clump are able to enrich clumps that have not yet formed their stars, resulting in intrinsic metallicity spreads once the clumps merge to form the final cluster. The model does a good job of matching the observed $[\mathrm{Fe} / \mathrm{H}]$ spreads of MW globular clusters. An open question is whether the same model can predict metallicity spreads of globular clusters formed in different environments. In this context, LMC globular clusters like NGC 1978 with metallicity spreads provide a very useful test.

We have run a sequence of models using the GCZCSE code (Bailin 2018b) that results in globular clusters with similar values to those obtained for NGC 1978 (Figure 4). The observed values of $[\mathrm{Fe} / \mathrm{H}]=-0.38 \pm 0.02$ and $W=0.035 \pm 0.019$ are fully consistent with the Bailin (2018a) model (panel (a)), demonstrating that clumpy self-enrichment is a viable mechanism for producing metallicity spreads in LMC clusters.

However, the metallicity spread in the models is a strong function of metallicity (panel (a)); though, Bailin (2018a) obtained models with $W$ as high as 0.40 for typical metal-poor MW clusters, at $[\mathrm{Fe} / \mathrm{H}] \approx-0.38$, the models saturate near $W \sim 0.08$. This is because spreads in $[\mathrm{Fe} / \mathrm{H}]$ correspond to the relative variation in metallicity: self-enrichment causes a fixed spread in the absolute metal mass fraction $Z$, but that spread becomes less and less noticeable as the magnitude of $Z$ increases. Put another way, a much more massive protocluster potential well is required to contain sufficient supernova ejecta to achieve a given value of $W$ at high metallicity. In panel (b), the initial globular cluster stellar mass of the same models is shown, along with the current observed stellar mass of NGC 1978 (open circle). The best-fit model from panel (a) requires the cluster to have had an initial mass $\log M_{\text {init }}=6.73$ (square), a factor of 18 more than the current mass. In other words, this model is only viable if mass loss has stripped nearly 95\% of its initial mass! The model with the minimum mass that passes through the error ellipse of the observations has an initial cluster mass of $\log M_{\text {init }}=6.47$ (triangle), still nearly 10 times larger than its current mass.

Globular clusters do indeed experience significant mass loss during their lives, both due to stellar evolution effects and tidal stripping. Balbinot \& Gieles (2018) modeled the evolution of over $150 \mathrm{MW}$ globular clusters, and found a mean mass ratio of 3 between the initial and final cluster masses (filled circle), with 
a)

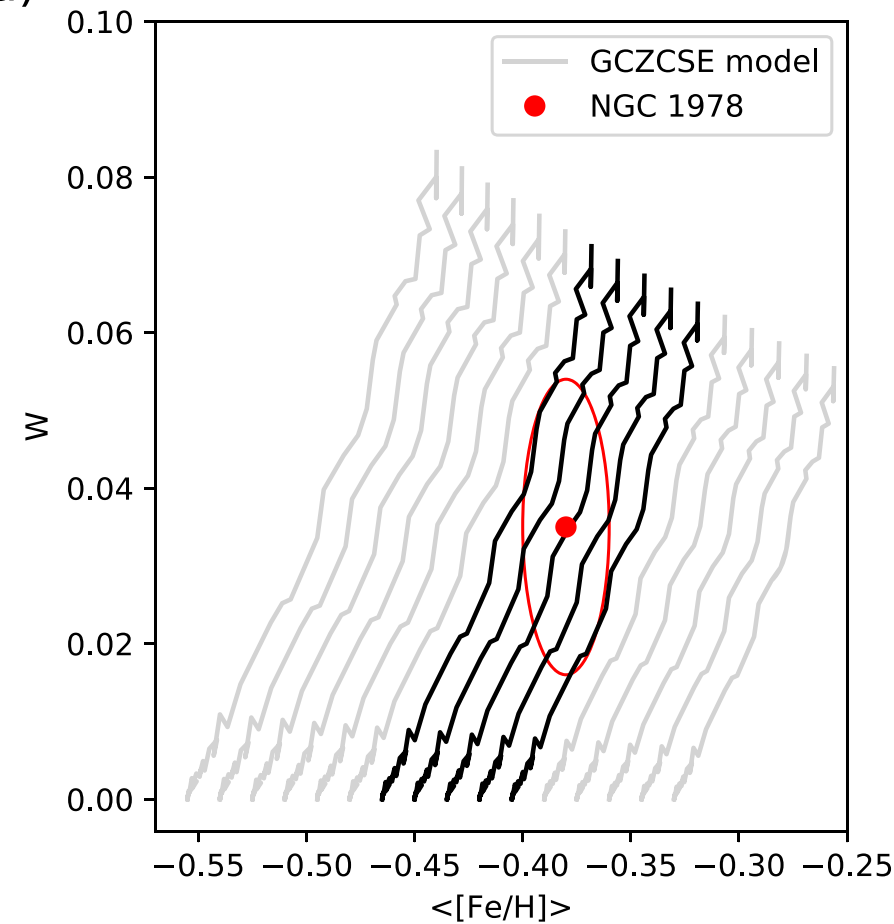

b)

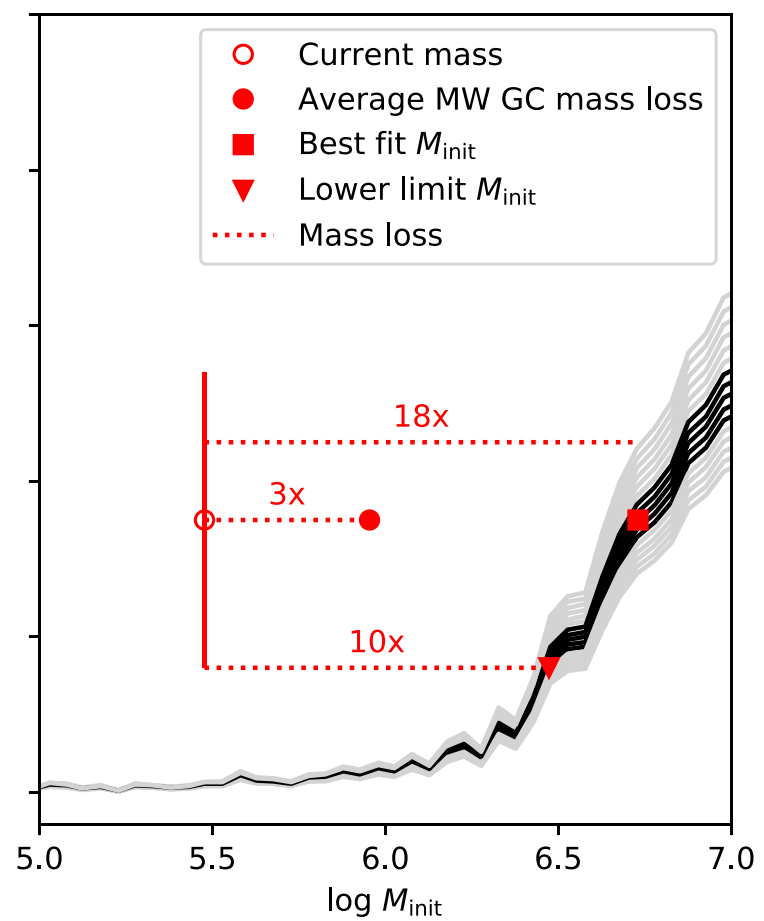

Figure 4. GCZCSE models of clumpy self-enrichment (Bailin 2018a). (a) Metallicity spread $W$ as a function of mean metallicity $\langle[\mathrm{Fe} / \mathrm{H}]\rangle$ for a sequence of models with different initial gas cloud metallicities. Our observations of NGC 1978 are presented in red, with the ellipse indicating the $1 \sigma$ uncertainties. Models highlighted in black are those that pass through the error ellipse. (b) The same models are shown as a function of the initial stellar mass of the globular cluster. The observed dispersion for NGC 1978 is shown as the open circle and error bar, plotted at its current stellar mass. The filled circle, square, and triangle indicate possible initial values for NGC 1978 if it has had mass loss typical of MW globular clusters (Balbinot \& Gieles 2018), if it lies on the best-fit model from panel (a), or if it lies on the model track with the minimum value of $M_{\text {init }}$ that passes through the error ellipse in panel (a) respectively. The labeled dotted lines indicate the required amount of mass loss in each case.

over $10 \%$ of clusters having mass ratios of at least 10 . Therefore, clusters with such extreme mass loss are unusual, but not unexpected. Furthermore, there is evidence that NGC 1978 is especially dynamically evolved. NGC 1978 lies at a LMC-centric radius of $3.5 \mathrm{kpc}$; Piatti \& Mackey (2018) found that LMC globular clusters within $5 \mathrm{kpc}$ of the center of the LMC have excesses of extratidal stars indicative of active tidal stripping. Moreover, they noted six MW clusters whose structural parameters matched those of the inner LMC clusters; these MW clusters are among the more highly stripped clusters in Balbinot \& Gieles (2018), with a mean mass-loss ratio of 6 , and extending to over 30 in the case of Terzan 4.

There are therefore five scenarios that can explain the observed spread in $[\mathrm{Fe} / \mathrm{H}]$ in NGC 1978:

1. The GCZCSE model is a good description of NGC 1978, which has lost at least $90 \%$ of its mass due to stellar evolution effects and tidal stripping.

2. NGC 1978 has experienced less extreme mass loss, but the parameters of the GCZCSE model depend on environment in such a way that LMC clusters experience more self-enrichment at a given initial cloud mass. The parameters that could increase $W$ at the relevant masses are increasing the mixing efficiency, decreasing the duration of star formation, making the stellar initial mass function more top-heavy, and/or decreasing the efficiency of star formation (Bailin 2018a). However, there is no obvious reason for any of these processes to have operated differently in the LMC than in the MW.
3. Some of the observed spread in $m_{1}$ is due to variations in $\mathrm{CN}$ abundance rather than $[\mathrm{Fe} / \mathrm{H}]$. If the initial cluster mass was only three times larger than the present mass, as for the average MW cluster, the predicted spread in $[\mathrm{Fe} / \mathrm{H}]$ is only $W \approx 0.002$, so in this scenario essentially all of the observed spread must be due to this effect.

4. An unrecognized source of photometric error is adding dispersion to the measurements of different stars in a way that somehow does not show up in our artificial star tests (we consider this unlikely, because we have used the empirical data to estimate the uncertainty).

5. The success of the GCZCSE model at describing MW globular clusters is a coincidence and is unrelated to the cause of metallicity spreads of globular clusters.

Scenario 1, which is consistent with the model, with observations of MW globular clusters, and with the structural parameters of inner LMC globular clusters, is our preferred explanation, but we cannot presently rule out the others.

\section{Conclusion}

With the aim of investigating whether MPs in massive LMC clusters exhibit spreads in the $[\mathrm{Fe} / \mathrm{H}]$ values, we made use of accurate Strömgren photometry to estimate individual $[\mathrm{Fe} / \mathrm{H}]$ values for RGB stars in NGC 1978. Particularly, we used the metallicity-sensitive index $m_{1}$, along with the high-dispersion spectroscopy-based metallicity calibration of Calamida et al. (2007), which provided us with $[\mathrm{Fe} / \mathrm{H}]$ values with an accuracy 
between 0.05 dex $\leqslant \sigma[\mathrm{Fe} / \mathrm{H}] \leqslant 0.25$ dex for previously selected bonafide cluster members.

The adopted uncertainties were thoroughly computed by taking into account every source of error, i.e., those coming from the photometry data set as well as those from the employed metallicity calibration. The photometric errors demanded extensive artificial star experiments in order to be reliably derived. In this sense, we mapped the photometric quality in terms of both the position of a star from the cluster's center and its brightness. When inspecting the different CMDs, a clear spread along the cluster's RGB is seen in CMDs that included the $m_{1}$ index, which is related to a spread in the $[\mathrm{Fe} / \mathrm{H}]$ values.

In order to quantify such a variation we performed a maximum likelihood analysis, from which we derived a dispersion of 0.035 dex with an error between 0.019 and $0.023 \mathrm{dex}$, depending on whether stars with individual $[\mathrm{Fe} / \mathrm{H}]$ errors smaller than $0.15 \mathrm{dex}$, or those located in the outer cluster's regions are considered. This outcome shows that NGC 1978 is, from the perspective of chemical anomalies in clusters with MPs, similar to the vast majority of MW globular clusters. On the other hand, we found that the resulting small metallicity spread is consistent with a cluster formation model where selfenrichment of chemical elements is produced by supernova ejecta, provided that a mass loss higher than $90 \%$ is assumed, because of stellar evolutionary effects and tidal stripping. Nevertheless, other scenarios should not be ruled out, such as a $\mathrm{CN}$ abundance variation reflected on the $[\mathrm{Fe} / \mathrm{H}]$ values, or less extreme mass loss with a more environmentally dependent selfenrichment model.

We thank the referee for the thorough reading of the manuscript and timely suggestions to improve it. Based on observations obtained at the Southern Astrophysical Research (SOAR) telescope, which is a joint project of the Ministério da Ciência, Tecnologia, Inovações e Comunicações (MCTIC) do Brasil, the U.S. National Optical Astronomy Observatory (NOAO), the University of North Carolina at Chapel Hill (UNC), and Michigan State University (MSU). Support for program HST-AR-13908.001-A was provided by NASA through a grant from the Space Telescope Science Institute, which is operated by the Association for Research in Astronomy, Inc., under NASA contract NAS 5-26555.

\section{ORCID iDs}

Andrés E. Piatti 1 i https://orcid.org/0000-0002-8679-0589

Jeremy Bailin (i) https://orcid.org/0000-0001-6380-010X

\section{References}

Bailin, J. 2018a, ApJ, 863, 99

Bailin, J. 2018b, GCZCSE, v.1.0.0, Zenodo, doi:10.5281/zenodo.1253347

Balbinot, E., \& Gieles, M. 2018, MNRAS, 474, 2479

Bekki, K. 2017, MNRAS, 469, 2933

Bekki, K., \& Tsujimoto, T. 2016, ApJ, 831, 70

Bressan, A., Marigo, P., Girardi, L., et al. 2012, MNRAS, 427, 127

Calamida, A., Bono, G., Stetson, P. B., et al. 2007, ApJ, 670, 400

Carretta, E., Bragaglia, A., Gratton, R., D’Orazi, V., \& Lucatello, S. 2009, A\&A, 508, 695

Crawford, D. L., \& Mandwewala, N. 1976, PASP, 88, 917

Davidge, T. J. 2018, ApJ, 856, 129

Ferraro, F. R., Mucciarelli, A., Carretta, E., \& Origlia, L. 2006, ApJL, 645, L33

Frank, M. J., Koch, A., Feltzing, S., et al. 2015, A\&A, 581, A72

Geisler, D., Piatti, A. E., Bica, E., \& Clariá, J. J. 2003, MNRAS, 341, 771

Gieles, M., Charbonnel, C., Krause, M. G. H., et al. 2018, MNRAS, 478, 2461

Goudfrooij, P., Girardi, L., Kozhurina-Platais, V., et al. 2014, ApJ, 797, 35

Hauck, B., \& Mermilliod, M. 1998, A\&AS, 129, 431

Kamath, D., Wood, P. R., Soszyński, I., \& Lebzelter, T. 2010, MNRAS, 408, 522

Kim, J. J., \& Lee, Y.-W. 2018, ApJ, 869, 35

Lederer, M. T., Lebzelter, T., Cristallo, S., et al. 2009, A\&A, 502, 913

Lim, D., Hong, S., \& Lee, Y.-W. 2017, ApJ, 844, 14

Marino, A. F., Milone, A. P., Karakas, A. I., et al. 2015, MNRAS, 450, 815

Martocchia, S., Cabrera-Ziri, I., Lardo, C., et al. 2018a, MNRAS, 473, 2688

Martocchia, S., Niederhofer, F., Dalessandro, E., et al. 2018b, MNRAS, 477, 4696

Milone, A. P., Bedin, L. R., Piotto, G., \& Anderson, J. 2009, A\&A, 497, 755

Ordoñez, A. J., \& Sarajedini, A. 2015, AJ, 149, 201

Paunzen, E. 2015, A\&A, 580, A23

Piatti, A. E. 2018, AJ, 156, 206

Piatti, A. E., \& Bastian, N. 2016a, MNRAS, 463, 1632

Piatti, A. E., \& Bastian, N. 2016b, A\&A, 590, A50

Piatti, A. E., \& Cole, A. 2017, MNRAS, 470, L77

Piatti, A. E., \& Koch, A. 2018, ApJ, 867, 8

Piatti, A. E., \& Mackey, A. D. 2018, MNRAS, 478, 2164

Pryor, C., \& Meylan, G. 1993, in ASP Conf. Ser. 50, Structure and Dynamics of Globular Clusters, ed. S. G. Djorgovski \& G. Meylan (San Francisco, CA: ASP), 357

Stetson, P. B., Davis, L. E., \& Crabtree, D. R. 1990, in ASP Conf. Ser. 8, CCDs in Astronomy, ed. G. H. Jacoby (San Francisco, CA: ASP), 289

Walker, M. G., Mateo, M., Olszewski, E. W., et al. 2006, AJ, 131, 2114

Werchan, F., \& Zaritsky, D. 2011, AJ, 142, 48

Willman, B., \& Strader, J. 2012, AJ, 144, 76 